\title{
MEASUREMENT OF THE NONSTEADY FLOW FIELD IN THE OPENING OF A RESONATING CAVITY EXCITED BY GRAZING FLOW
}

\author{
H. R. Graf† AND W. W. Durgin \\ Department of Mechanical Engineering, Worcester Polytechnic Institute \\ Worcester, MA 01609,USA
}

(Received 20 August 1990 and in revised form 4 September 1992)

\begin{abstract}
Flow past the opening of a cavity can excite acoustic resonance. The periodic velocity field in the region of the cavity opening has been measured for various flow conditions and the vorticity distribution has been computed from the measured data using numerical differentiation. The results indicate that the shear layer rolls up into discrete vortices, which travel across the cavity opening. Two resonances were found. The first is characterized by a single vortex being present and results in the greatest amplitude. The second is characterized by the presence of two vortices which excite a distinct but lesser amplitude resonance. As the flow velocity changes, the position of these vortices shifts relative to the phase of the acoustic cycle. The timing of the interaction between the moving vortices and the acoustic particle velocity determines, to a large extent, the intensity of the excitation, and therefore also the oscillation amplitude. The measurements also indicate that the boundary layer upstream of the leading edge pulsates considerably.
\end{abstract}

\section{INTRODUCTION}

ANY DEEP CAVITY WITH SOLID WALLS can support acoustic resonance at the natural frequencies of the cavity. Flow across the opening of the cavity can excite strong oscillation at resonance. A good illustration of this effect is the tone that can be produced by blowing across the opening of a bottle.

While the bottle-experiment is a popular demonstration of this fluid dynamic phenomenon, there are also important engineering applications. Flow-induced cavity oscillations can cause intense noise with discrete frequencies. The high amplitude of the acoustic pressure in the cavity results in mechanical stresses in surronding structures and can cause fatigue failure. The resonating cavity also extracts energy from the flow, which can significantly increase the fluid dynamic loss coefficient or drag.

Situations where these problems occur are commonly found in piping systems, in valves, and in wheel-wells and bays of aircraft. Bruggeman (1987) reported cavity oscillations in a pipeline installation for natural gas, where closed side branches in a compressor station formed cavities. The acoustic pressure pulsations of up to 1.75 bar rms caused severe structural vibrations and made it necessary to avoid the operating condition where resonance occurred.

Furthermore, the effect is also of scientific interest. The mechanism of the excitation of acoustic resonance by a shear layer is not yet well understood and is of fundamental interest in the area of flow-induced vibration and acoustics.

$\dagger$ Present address: Sulzer Brothers Limited, Laboratory for Vibrations and Acoustics, CH-8401 Winterthur, Switzerland. 


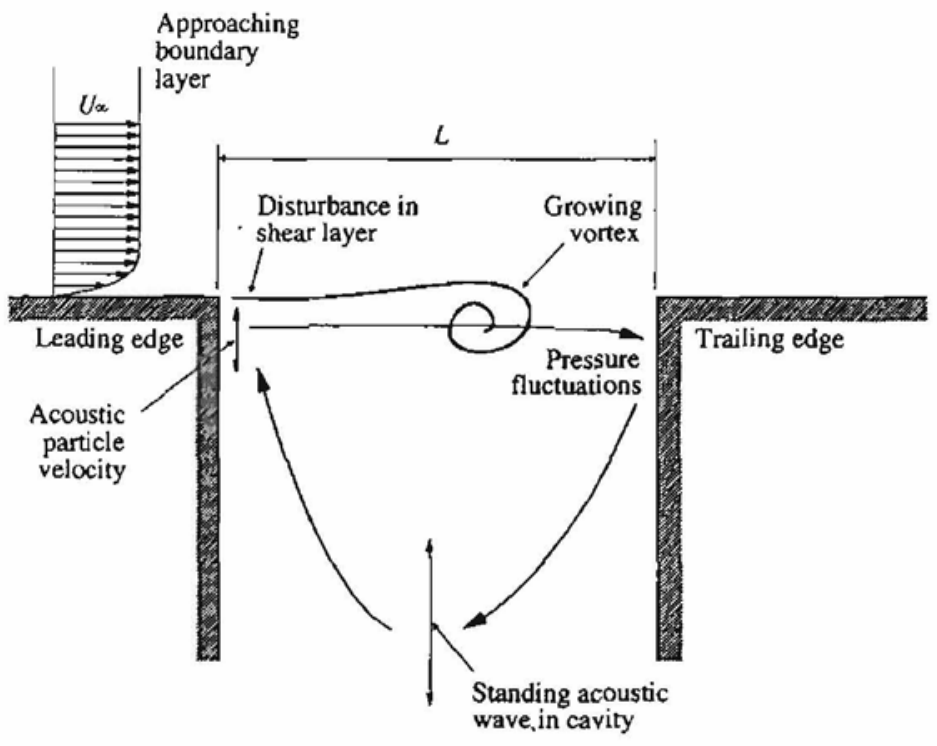

Figure 1. Feedback loop of self-excited cavity oscillation.

\subsection{Feedback Loop}

The excitation of the cavity oscillation is characterized by the feedback loop depicted in Figure 1. The grazing flow forms an unstable shear layer in the cavity mouth. The acoustic standing wave in the cavity causes disturbances in the shear layer near the upstream edge. These disturbances grow rapidly and cause the vorticity in the shear layer to roll into large-scale vortices. When the vortices travel across the opening, they cause pressure fluctuations, which drive the acoustic oscillation. The oscillation, in turn, triggers the periodic formation of new vortices.

The overall gain in this feedback loop and the losses due to radiation of acoustic power determine the amplitude of the acoustic oscillation.

Since the acoustic standing wave plays an essential part in the feedback loop, Rockwell \& Naudascher (1978) termed this type of flow induced cavity oscillation "fluid resonant".

\subsection{Dimensionless Parameters}

The flow condition is characterized by the reduced velocity, $U_{r}$, which is defined as the free-stream velocity, $U_{\infty}$, divided by the streamwise dimension of the cavity, $L$, and the acoustic natural frequency of the cavity, $f_{n}$.

The amplitude of the flow-induced oscillation is quantified by $V$, which is the root-mean-square of the acoustic particle velocity into and out of the cavity, divided by the free-stream velocity. Since it is difficult to measure the acoustic velocity directly, the amplitude of the acoustic pressure at the closed end was measured instead. The acoustic particle velocity at the opening of the cavity was then computed from the pressure data, using a model for the standing wave in the cavity (Graf 1989).

Figure 2 shows the oscillation amplitude as a function of the reduced velocity. Strong resonance is observed for two flow conditions, corresponding to single- and doublevortex modes.

In the single-vortex mode, only one vortex populates the cavity at any given time. At $U_{r}=3 \cdot 2$, the acoustic pressure in the cavity is very high and can exceed the dynamic 


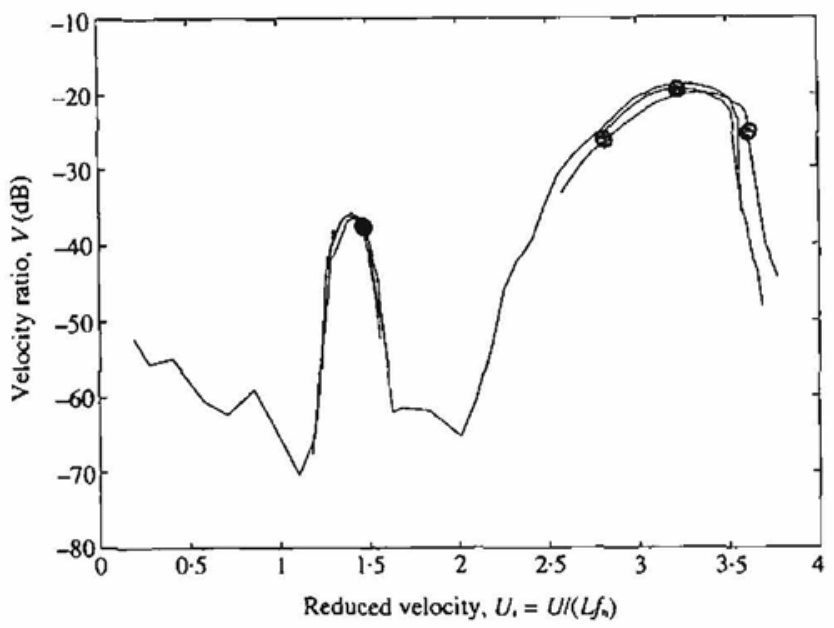

Figure 2. Oscillation amplitude as a function of the reduced velocity. $V=$ Root-mean-square of the acoustic velocity at the cavity mouth divided by $U_{\infty}$. The symbols indicate flow conditions for the detailed velocity measurements.

pressure of the external flow. The amplitude of the acoustic velocity into and out of the cavity is up to $15 \%$ of the free-stream velocity. The double-vortex mode occurs at $U_{r} \simeq 1 \cdot 5$. Here, two vortices are in the cavity opening simultaneously. The amplitude of the oscillation, however, is not as strong.

\subsection{Theoretical Models of Flow-Excited Cavity Resonance}

In the classical approach to this problem, the growth of small, wave-like disturbances in the shear layer, which are induced by the acoustic oscillation, have been modelled using stability theory. The disturbances then gave rise to an acoustic source which, in turn, excited the acoustic resonance, thus completing the feedback loop (Rockwell \& Naudascher 1978; Elder 1978).

Nelson et al. $(1981,1983)$ approached the problem of flow-excited resonance in a Helmholtz resonator by investigating the physics of the flow-acoustic interaction in terms of momentum and energy exchanges occurring in the fluid itself. This alternative approach is supported by the fact that the disturbances in the shear layer are large and cannot be modelled appropriately by a linear or weakly nonlinear stability theory.

To support this theory, Nelson et al. (1981) carried out measurements of the flow field in the neck of a Helmholtz resonator excited to peak amplitude. With Laser-Doppler Velocimetry they measured the average velocity field and the magnitude and phase of the first harmonic. However, only part of the resonator neck was accessible for the laser beams; information about the velocity field in the important region near the upstream edge could not be obtained.

Bruggeman (1987) and Bruggeman et al. (1989) investigated flow-excited resonance in closed side branches of gas pipelines. Flow in the opening of the side branch was modelled with discrete vortices and identified the mechanism by which the moving vortices excite the acoustic oscillation. This model showed that the excitation of the oscillation is determined to a large extent by the position of the vortex during the acoustic cycle and by the distribution of the vorticity.

The excitation of the acoustic oscillation takes place in the region of the cavity opening. In the experiments presented here, the flow field in this region was measured 
in detail. Various flow conditions were analysed in order to better understand the excitation mechanism.

\section{EXPERIMENTAL SET-UP}

\subsection{FLOW MODEL}

Experiments were conducted using an open circuit wind tunnel with the cavity attached to the test section. The apparatus was designed to produce two-dimensional flow. The streamwise dimension of the cavity opening, which is the characteristic dimension, was $L=65.5 \mathrm{~mm}$. The edges of the opening were left sharp and two acrylic sheets, spaced $178 \mathrm{~mm}$ apart, formed the sidewalls of the flow channel and of the cavity. These $9 \mathrm{~mm}$ thick cast acrylic sheets also provided optical access for Laser Doppler Velocimetry. At the lower end, the cavity was closed by a piston. The cross-section of the cavity was slightly enlarged near the closed end, as depicted in Figure 3, in order to increase the resonant frequencies of the higher acoustic modes in the cavity and, thereby, reduce their interaction with the fundamental mode. The first natural frequency of the cavity was measured at $242 \mathrm{~Hz}$, the second was observed at $825 \mathrm{~Hz}$.

The acoustic pressure at the closed end of the cavity was measured using a piezo-electric pressure transducer located at the center of the closed end. This pressure signal was used to determine the amplitude, frequency, and phase of the cavity oscillation.

To obtain a well-defined turbulent boundary layer, a sheet of sandpaper was attached to the wall of the wind tunnel upstream of the test section. The momentum

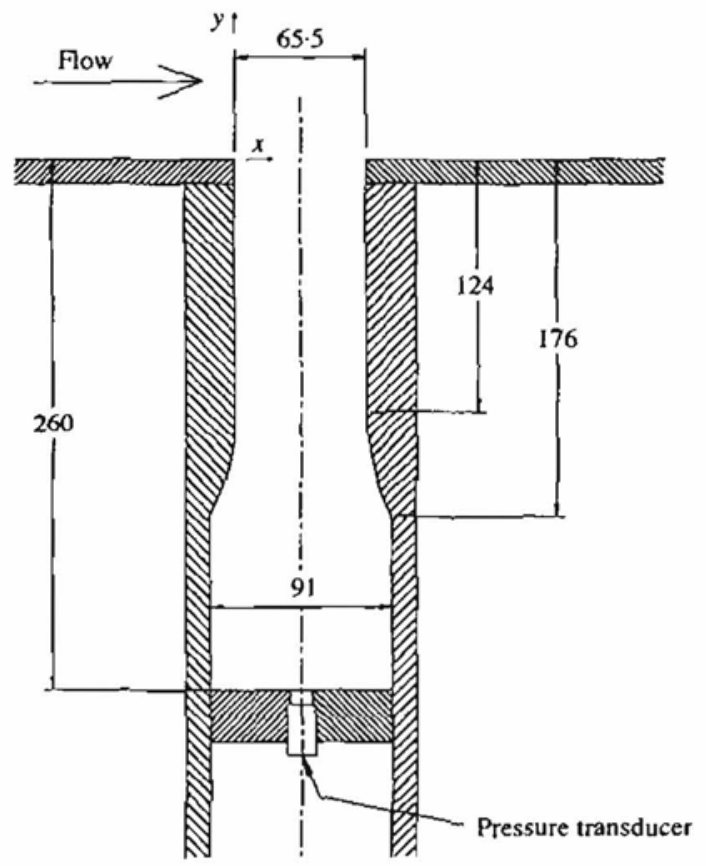

Figure 3. Cavity geometry. Dimensions are in $\mathrm{mm}$. 
thickness of the boundary layer at the upstream edge of the cavity opening was approximately $6.4 \mathrm{~mm}$ or $10 \%$ of the cavity opening.

\subsection{Laser Doppler Velocimetry System}

The periodic velocity field in the region of the cavity mouth was measured using Laser Doppler Velocimetry. The LDV system operated in the backscatter mode and consisted of standard optical components including an Argon-ion laser, a frequency shifter, and a $2 \cdot 27 \times$ beam expander. The focal length of the transmitting lens was $600 \mathrm{~mm}$. The output power of the laser was usually adjusted to $500 \mathrm{~mW}$.

The system measured only one velocity component. Therefore, after measuring the velocity in $x$-direction, the optics were rotated by $90^{\circ}$ in order to measure the $y$-component of the velocity.

The seeding particles required for LDV were produced from propylene glycol in an evaporation/condensation type smoke generator. The seeding particles were introduced into the flow in front of the intake section of the wind tunnel.

The Doppler signals, which were generated when a seeding particle travelled through the measuring volume, were analysed and converted to digital data by a counter-type signal processor. The digital data were then transferred to a specially designed computer interface. Here, the velocity data was complemented by the phase information, indicating the time in the acoustic cycle at which the velocity sample occurred. This time information was provided by a digital clock, which was reset when the pressure at the closed end of the cavity was zero and increasing. The clock, therefore, measured the time since the beginning of the current acoustic cycle. The accuracy of this time (or phase) information was about $4^{\circ}$ or $1 \%$ of an acoustic cycle. The complete data set was then transferred to a personal computer for analysis.

After about 5000 velocity samples were taken, the data were analysed using conditional sampling and fast Fourier transforms. The results of this analysis were the harmonic components of the averaged velocity waveform. The first four to six harmonics were significant. Components at higher frequencies were primarily due to turbulence and non-periodic velocity fluctuations and were therefore ignored. The method used to analyse the data were described in detail in earlier publications (Graf $e t$ al. 1988, 1989). Using this approach, the periodic velocity distribution was sampled at measuring points covering the region of the cavity mouth with a dense grid.

\section{RESULTS}

\subsection{Single Vortex Mode}

Figure 4 shows the measured velocity field at resonance in the single vortex mode. The velocity is plotted for ten different phase angles, $\varphi$, in the acoustic cycle. (The phase $\varphi$ is zero when the velocity into the cavity is maximum. The acoustic pressure in the cavity is then zero and increasing.)

The vortex in the shear layer forms at the upstream edge when air starts flowing into the cavity $(\varphi \simeq 0.8 \times 2 \pi)$. Subsequently, the vortex grows in strength, travels downstream, and reaches the trailing edge about one acoustic cycle later.

The question whether a Kutta condition must be satisfied at the upstream edge has been a controversial issue for a long time, especially in the case of unsteady flow (Crighton 1981; Michalke 1984). Compliance with the Kutta condition was found to be 
H. R. GRAF AND W. W. DURGIN
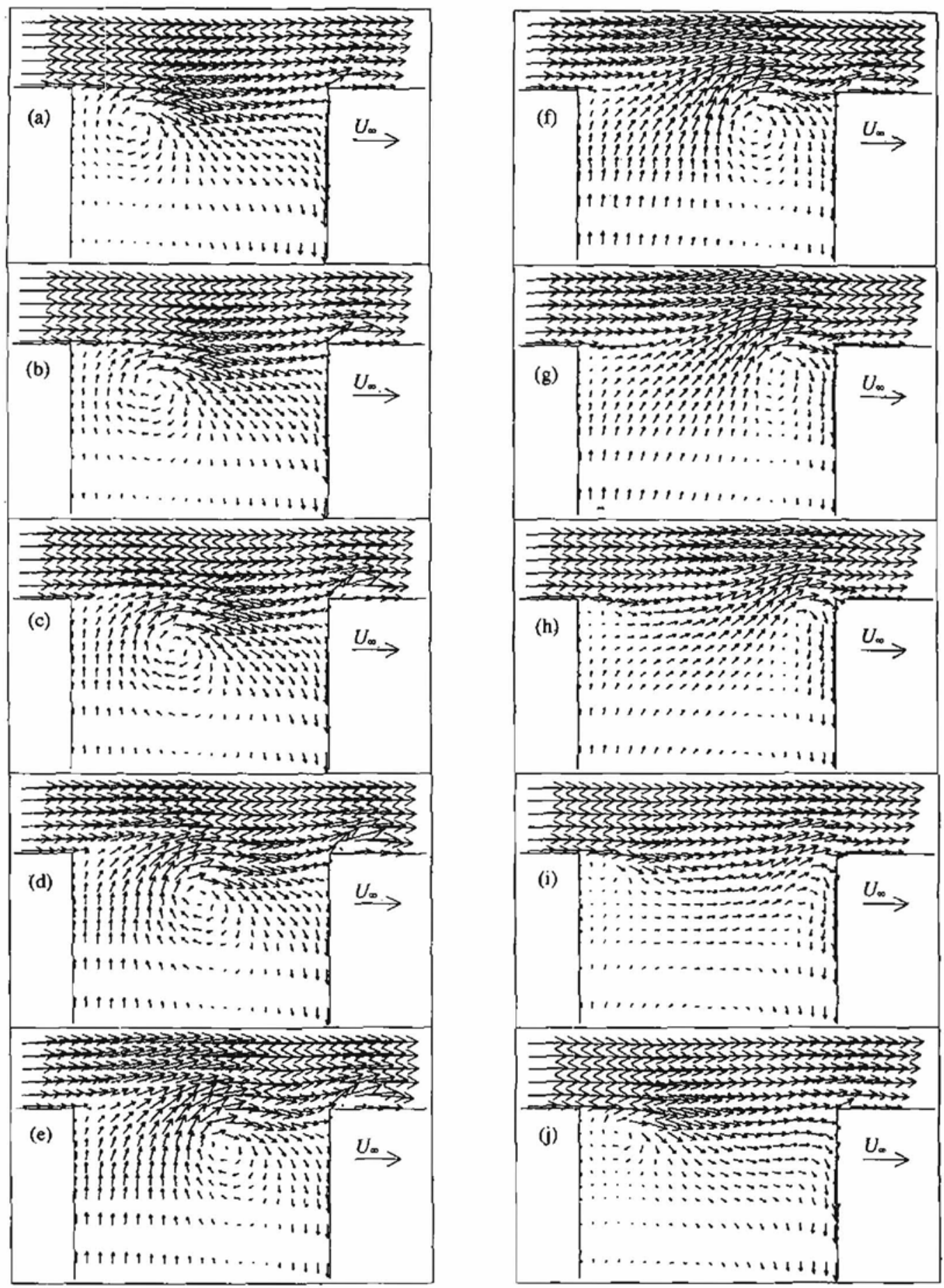

Figure 4. Velocity field for $U_{r}=3.21, V=0.1$ : (a) $\varphi=0$; (b) $\varphi=0.1 \times 2 \pi$; (c) $\varphi=0.2 \times 2 \pi$; (d) $\varphi=0.3 \times 2 \pi$; (e) $\varphi=0.4 \times 2 \pi$; (f) $\varphi=0.5 \times 2 \pi$; (g) $\varphi=0.6 \times 2 \pi$; (h) $\varphi=0.7 \times 2 \pi$; (i) $\varphi=0.8 \times 2 \pi$; (j) $\varphi=0.9 \times 2 \pi$. 
an important factor in determining the dynamics of the flow/acoustic interaction in the work of Nelson et al. (1983) and it was also a necessary assumption in the theoretical treatments presented by Howe (1979). Contrary to this, some theoretical investigations (e.g., Orszag \& Crow 1970) lead to a "no Kutta condition" for an infinitely thin splitter plate, resulting in a singularity in the velocity field and the shear layer leaves the edge with an infinite slope.

Our velocity measurements indicate that the flow immediately downstream of the edge remains parallel to the channel wall or is slightly deflected towards the external flow. No indication of a singularity in the velocity field is observed near the edge. The flow velocity along the inner wall of the upstream corner decreases towards the separation point and appears to vanish at the edge. These observations indicate that the full Kutta condition is satisfied. Notice, however, that the flow field near the upstream edge was not mapped with a very detailed resolution and that the approaching turbulent boundary layer is much thicker than in most investigations of the Kutta condition.

During part of the cycle $(\varphi=-0.1 \times 2 \pi, \ldots, 0.5 \times 2 \pi)$ a small region of separated flow forms just downstream of the trailing edge due to edge separation; the flow reattaches when the following vortex approaches, inducing a change in flow direction at the edge. A similar phenomenon of periodic separation at the impingement edge is an important feature in sheartone and edgetone excitation (Elder et al. 1982; Ziada \& Rockwell 1982) where upstream feedback is a fluid-dynamic mechanism. This sheartone mechanism, however, is not considered important in these experiments, since the cavity oscillations are here excited by a much stronger fluid-resonant feedback mechanism.

The nonsteady vorticity field is plotted in Figure 5 as a three-dimensional surface. The vorticity distribution was computed from the measured velocity by numerical differentiation. The central difference method was employed inside the flow field; one-sided difference schemes were used at the boundaries.

At phase $\varphi \approx 0.6 \times 2 \pi$ it can be seen that vorticity coming from the approaching boundary layer starts accumulating into a large vortex just downstream of the leading edge. When $\varphi \simeq 0.8 \times 2 \pi$ the vortex starts travelling downstream across the cavity opening. About one acoustic cycle later this vortex impinges on the downstream edge and is cut in half; one part of the vortex moves into the cavity along the downstream wall. The other portion of the vortex appears to be trapped and reinforced in the separation region downstream of the trailing edge until the separated flow reattaches at phase $\varphi=0.6 \times 2 \pi$.

The behaviour at the downstream edge is somewhat different from that observed by Nelson et al. (1981), where the entire vortex appeared to be pushed out of the cavity. In a recent flow visualization study, Ziada (1992) observed that at low and moderate pulsation amplitudes the vortex impinges on the downstream edge as in Figure 4; at high amplitudes $(V>0.3)$, however, the entire vortex is expelled from the cavity.

After the vortex is fully developed $(\varphi \geq 0.5 \times 2 \pi)$, most of the vorticity is concentrated in the discrete vortex-blob; the remaining area of the cavity opening is essentially free of vorticity.

Some sources of error should be kept in mind. They are inherent to the finite difference approximation used to calculate the vorticity field. First of all, small errors in the velocity data are amplified when the differences between adjacent points of the velocity grid are computed and divided by the grid spacing. With an estimated maximum error in the velocity data of $0.01 U_{\infty}$, the error in computed vorticity could be as high as $0 \cdot 2 U_{\infty} / L$. Some dither which might be associated with this effect can be observed in the free-stream region in Figures 5 and 7. Furthermore, sharp peaks of 

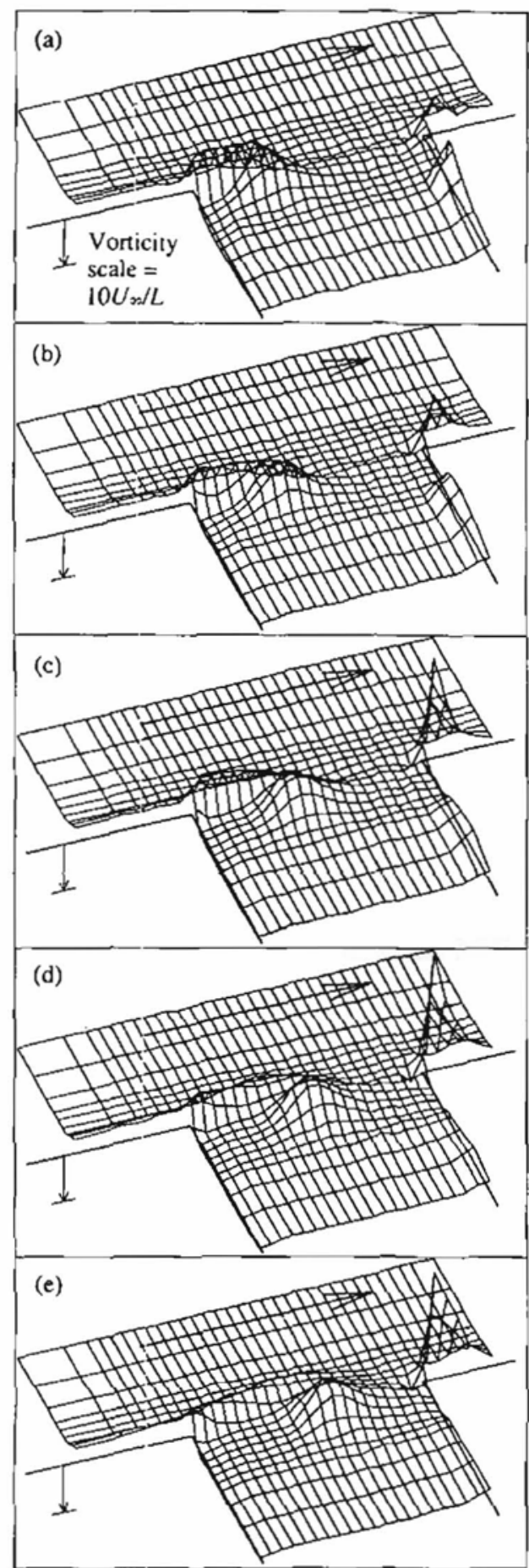
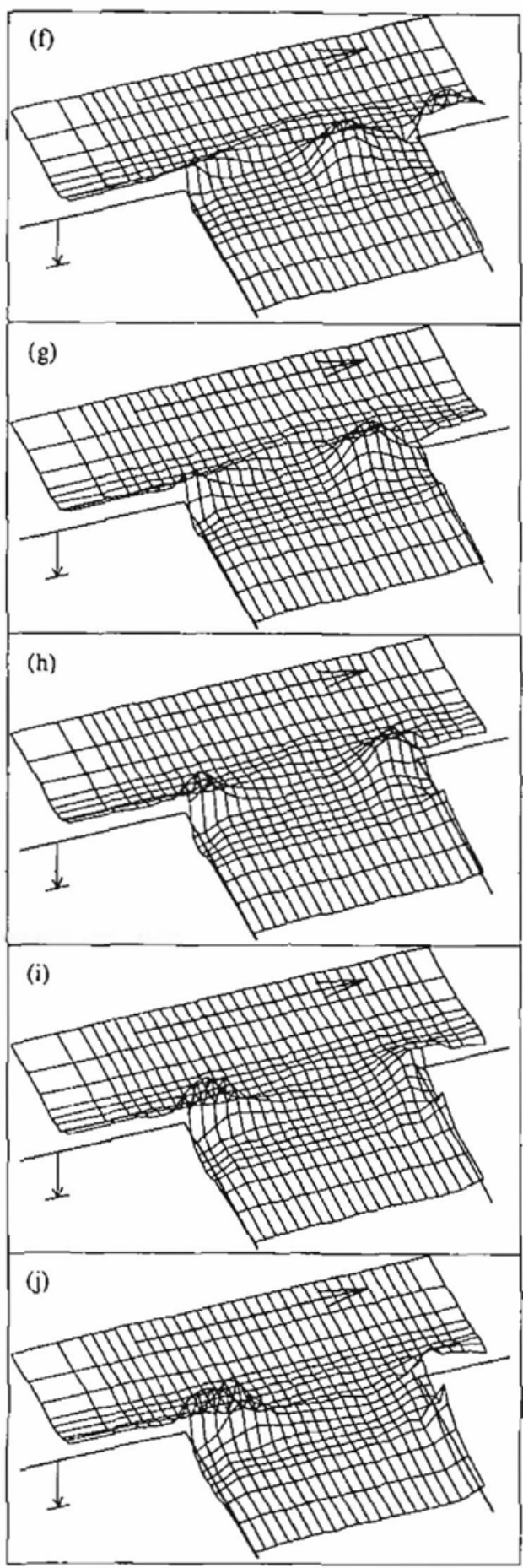

Figure 5. Vorticity field for $U_{r}=3.21, V=0.1$. (a) $\varphi=0$; (b) $\varphi=0.1 \times 2 \pi$; (c) $\varphi=0.2 \times 2 \pi$; (d) $\varphi=0.3 \times 2 \pi$; (e) $\varphi=0.4 \times 2 \pi$; (f) $\varphi=0.5 \times 2 \pi$; (g) $\varphi=0.6 \times 2 \pi$; (h) $\varphi=0.7 \times 2 \pi$; (i) $\varphi=0.8 \times 2 \pi$; (j) $\varphi=0.9 \times 2 \pi$. 
NONSTEADY FLOW ABOVE A RESONATING CAVITY
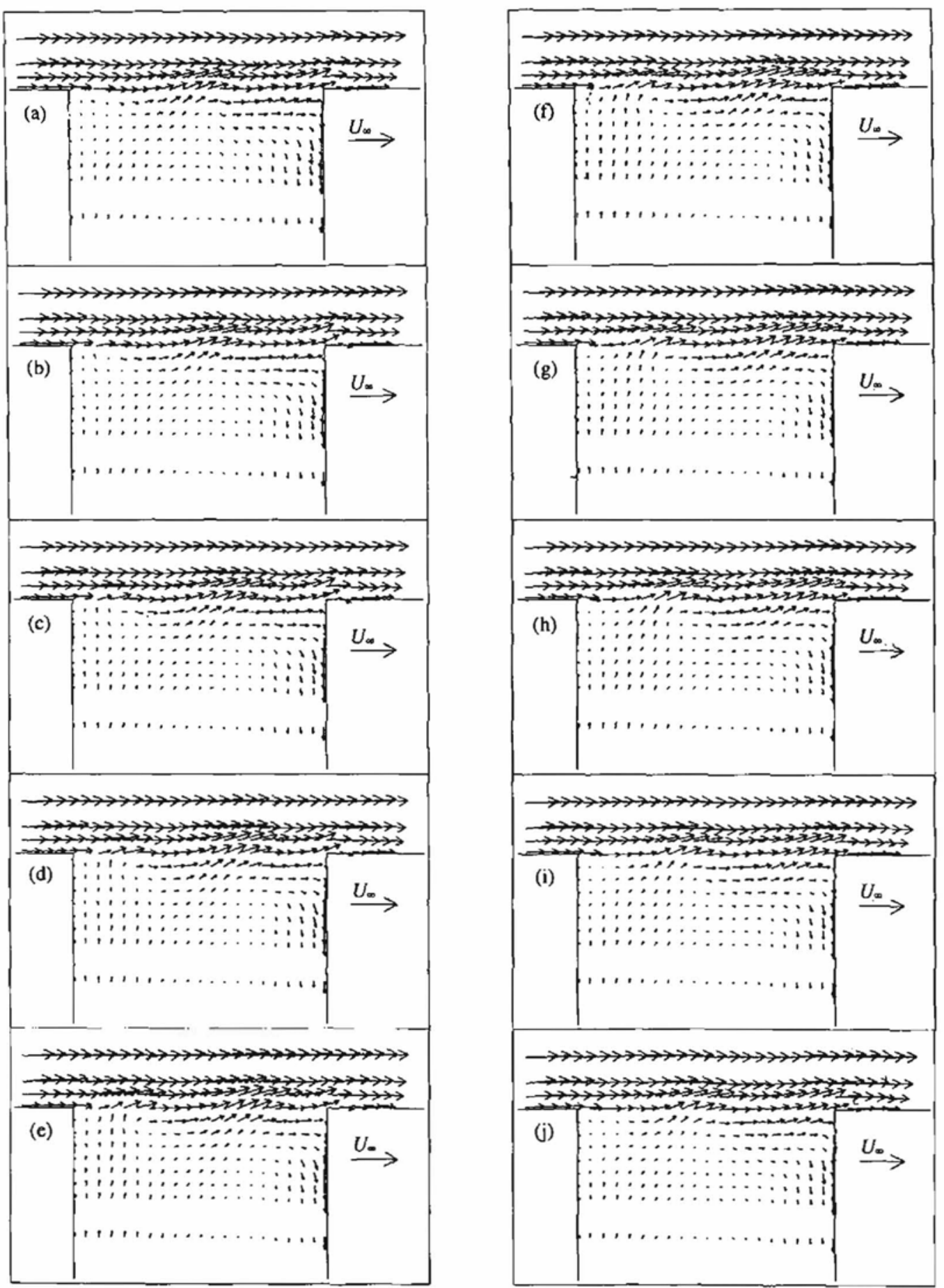

Figure 6. Velocity ficld for $U_{y}=1.46, V=0.013$; (a) $\varphi=0$; (b) $\varphi=0.1 \times 2 \pi$; (c) $\varphi=0.2 \times 2 \pi$; (d) $\varphi=0.3 \times 2 \pi$; (e) $\varphi=0.4 \times 2 \pi$; (f) $\varphi=0.5 \times 2 \pi$; (g) $\varphi=0.6 \times 2 \pi$; (h) $\varphi=0.7 \times 2 \pi$; (i) $\varphi=0.8 \times 2 \pi$; (j) $=\varphi=0.9 \times 2 \pi$. 

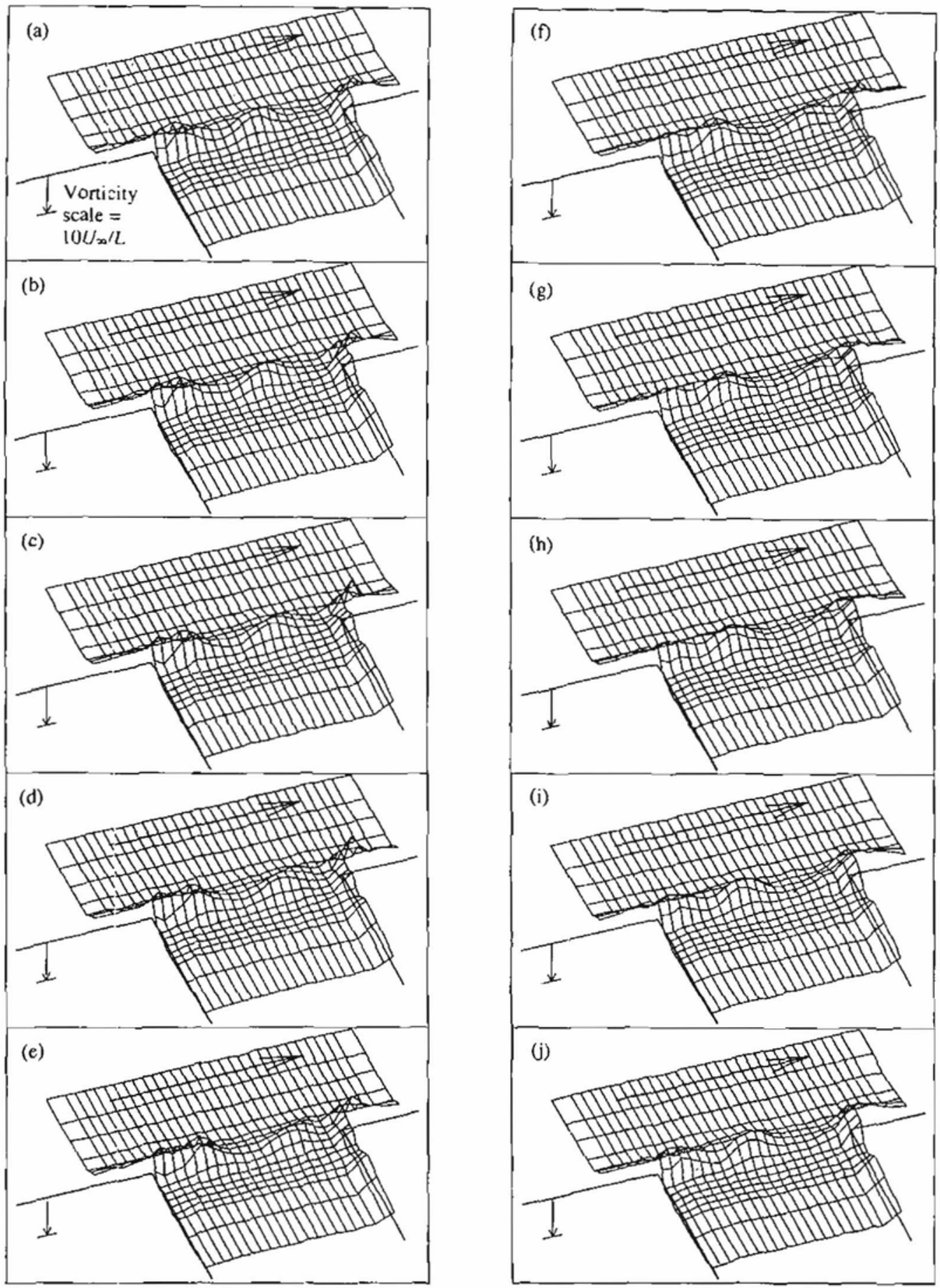

Figure 7. Vorticity field for $U_{r}=1.46, V=0.013$. (a) $\varphi=0$; (b) $\varphi=0.1 \times 2 \pi$; (c) $\varphi=0.2 \times 2 \pi$; (d) $\varphi=0.3 \times 2 \pi$; (e) $\varphi=0.4 \times 2 \pi$; (f) $\varphi=0.5 \times 2 \pi ;$ (g) $\varphi=0.6 \times 2 \pi ;$ (h) $\varphi=0.7 \times 2 \pi ;$ (i) $=\varphi=0.8 \times 2 \pi ;(\mathrm{j})$ $\varphi=0.9 \times 2 \pi$. 
vorticity are not represented correctly. They are distributed over one or more grid cells, depending on the method used. The extremely high vorticity in the laminar sublayer adjacent to the wall, for example, would not be determined correctly. For this reason no attempt was made to plot the vorticity in the wall regions. The data points closest to the wall are $0.5 \mathrm{~mm}$ away from the surface.

\subsection{Double-Vortex Mode}

In the double-vortex mode the oscillation is not as strong and the vortices are not as well defined. However, in Figure 6, two regions of distortion in the shear layer, which correspond to vorticity concentrations, can still be recognized.

In the vorticity plot of Figure 7 the two vortices in the cavity mouth appear as two discrete "hills" containing fluid with elevated levels of vorticity. These vortices also form at the leading edge and travel downstream. However, they travel only half the distance to the trailing edge during one acoustic cycle and reach the trailing edge after approximately two cycles. Consequently, two vortices are in the shear layer simultaneously. Note that still only one new vortex is formed in each period.

\subsection{Vortex Posmion}

In the single-vortex mode the velocity field was also measured at reduced velocities below and above resonance. Figure 2 shows the conditions for all four measurements. The location of the vortex core as a function of the phase in the acoustic cycle was determined from the vorticity plots, as shown in Figure 8.

As noted in Section 3.1, at resonance in the single-vortex mode the vortex leaves the upstream edge at about $\varphi=-0.25 \times 2 \pi$, i.e. when the direction of the acoustic velocity changes so that air starts flowing into the cavity. Subsequently, the vortex travels across the cavity mouth at a nearly constant velocity. Expressed as distance travelled per acoustic cycle, the convective velocity of the vortex is $U_{c}=0.9 \mathrm{~L} /$ cycle. This corresponds to $U_{c} / U_{\infty} \approx 0.3$, which matches the Strouhal number approximately.

This result agrees with the simple concentrated vortex model by Bruggeman et al. (1989), which also predicted that the acoustic power generated by the vortex is maximum when $U_{c} / U_{\infty}=S$. The model further suggests that the generation of acoustic power is highly sensitive to the timing between the acoustic oscillation and the interaction between the vortex and the downstream edge.

Above resonance $\left(U_{r}=3.6\right)$ the propagation velocity of the vortex remains unchanged; however, the vortex position is approximately $0 \cdot 1 \mathrm{~L}$ further downstream for corresponding phase angles, $\varphi$. Due to the different vortex position, the timing of the interaction with the trailing edge is altered and the transfer of acoustic power is consequently reduced.

Below resonance $\left(U_{r}=2 \cdot 81\right)$, a different mechanism must be responsible for the reduction of the amplitude, since the vortex location is essentially the same as at resonance. A flow visualization study currently under way (Ziada 1992) indicates that vortex propagation during the acoustic cycle is not only a function of Strouhal number but also varies considerably with pulsation amplitude. It appears that the oscillation amplitude for $U_{r}$ below resonance is controlled by this phenomenon.

In the double-vortex mode the convective velocity of the vortices is also $U_{c}=0.3 U_{\infty}$. However, due to the higher Strouhal number the distance travelled per acoustic cycle is only $0.45 L$ approximately. Still, the vortices leave the upstream edge and arrive at the downstream edge at about the same phase, $\varphi$, as for resonance in the single vortex 


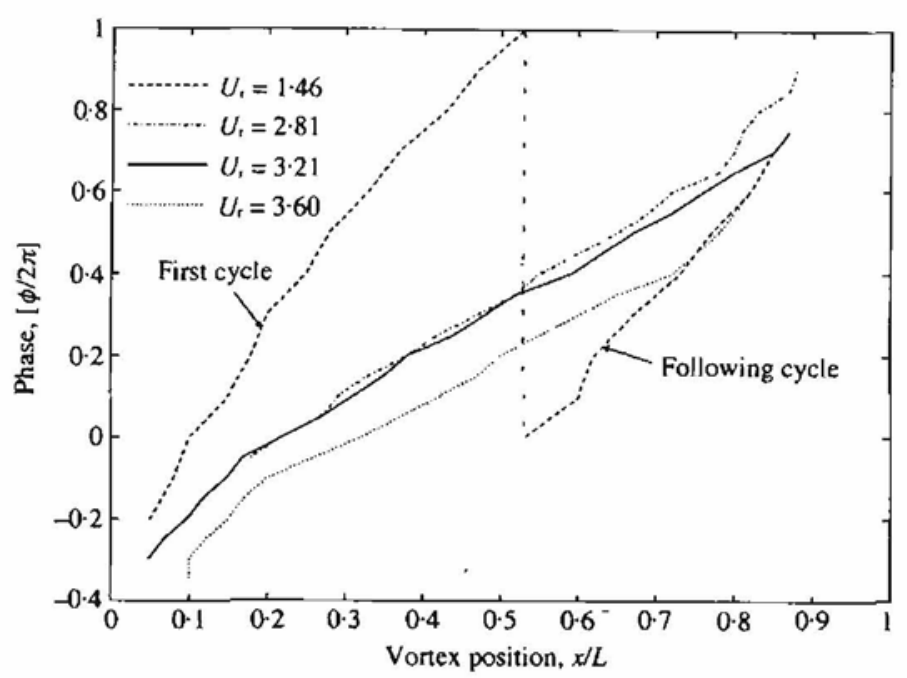

Figure 8. $x$-location of the vortex in the cavity opening as a function of the phase in the acoustic cycle. Note that for $U_{r}=1.46$ the vortex needs more than two cycles to travel across the opening.

mode. This further supports the hypothesis that the timing of the interaction of the vortices with the upstream and downstream edges is important and that the position of the vortex inside the opening of the cavity is less crucial.

The vortex propagation of Figure 8 agrees fairly well with experimental results for the single-vortex-mode reported by Bruggeman (1987). The propagation velocity of the vortex in the present investigation is about $0.3 U_{\infty}$ compared to $0.4 U_{\infty}$ in Bruggeman's (1987) experiments. The Strouhal numbers at resonance are $\mathrm{St}=0.32$ and $\mathrm{St}=0.4$, respectively. This discrepancy is probably due to the different thickness of the approaching boundary layer, which in this case was $\theta=0 \cdot 1 L$, whereas Bruggeman reported $\theta=0.005 L$.

The effect of the boundary layer thickness was first investigated by DeMetz \& Farabee (1975) and was also observed by Elder (1978 and Elder et al. 1982). It seems that the propagation velocity of the vortex is not directly a function of $U_{\infty}$, but rather proportional to some characteristic flow velocity in the region of the cavity mouth. If the boundary layer is relatively thick compared to $L$ this characteristic velocity becomes smaller than $U_{\infty}$. Thus, the Strouhal number at resonance is smaller for thick boundary layers, since St is still based on $U_{\infty}$.

\subsection{Boundary Layer Pulsations}

The velocity measurements also indicate that the approaching flow is pulsating considerably. Figure 9 shows the boundary layer just upstream of the leading edge. The velocity profile is plotted for four different phase angles.

When air is flowing into the cavity $(\varphi=0)$, the approaching fluid is accelerated and the velocity profile is full. With this velocity profile the rate of vorticity shedding at the edge is high, thus promoting the formation of the large-scale vortex. (Note that the shedding rate of vorticity at the upstream edge is proportional to the square of the velocity above the wall.)

Half an acoustic cycle later $(\varphi=\pi)$ the profile is retarded due the adverse pressure gradient. Here the shedding rate of vorticity at the edge is about $60 \%$ smaller than for $\varphi=0$. 


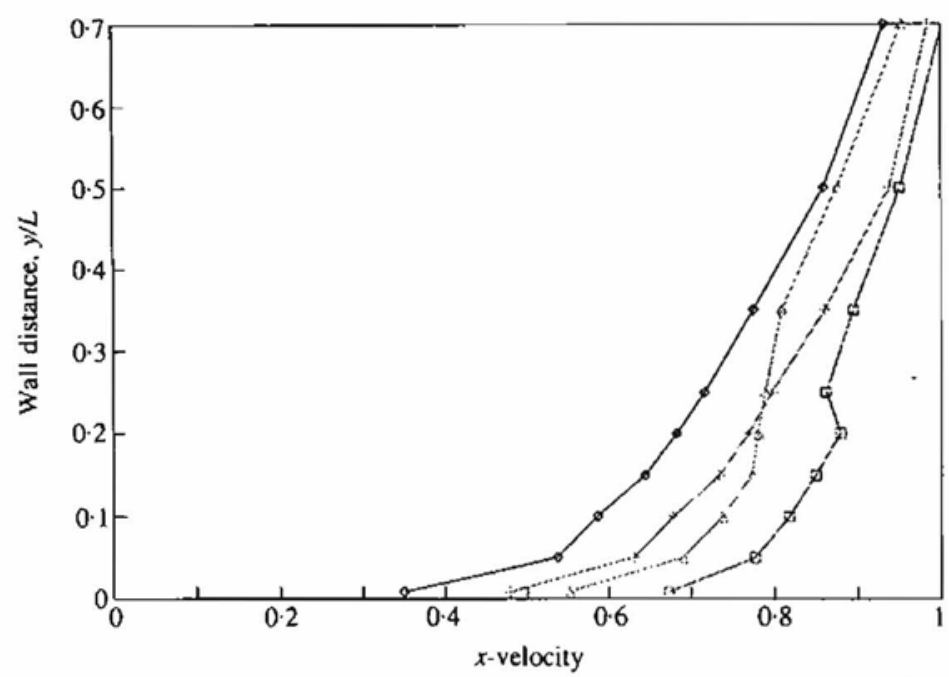

Figure 9. Nonsteady boundary layer profiles at $x=-0 \cdot 1 L$ for different phase angles in the acoustic cycle: $\square, \varphi=0 ;+, \varphi=\frac{1}{2} \pi ; \diamond, \varphi=\pi ; \Delta, \varphi=\frac{3}{2} \pi$.

Close to the wall, the difference between maximum and minimum velocity is about $30 \%$ of the free-stream velocity. Figure 9 indicates that the phase of the velocity fluctuations changes at about $y=0 \cdot 25 \mathrm{~L}$. This feature is often observed in pulsating turbulent boundary layers (Cook 1986).

In an earlier computer simulation of the nonsteady flow, Graf (1989) has demonstrated that the velocity pulsations of the boundary layer and the related fluctuations in the release of vorticity can play a significant role in the triggering of the roll-up of the shear layer.

\section{CONCLUSIONS}

The experiments presented in this paper provide detailed information about the nature of the nonsteady flow field in the region of the opening of a resonating cavity. At resonance in the single-vortex mode most of the vorticity in the shear layer is concentrated in a discrete free-moving vortex. In the double-vortex mode two regions of increased vorticity are clearly visible in the cavity mouth.

The comparison of the behavior of the vortices for different flow conditions reveals that the position of the vortex shifts relative to the acoustic oscillation when the reduced velocity is above the resonance condition. This decreases the acoustic power transferred from the flow to the acoustic oscillation.

The boundary layer upstream of the cavity pulsates considerably due to the acoustic oscillation. This contributes to the periodic formation of the large scale vortices down-stream of the leading edge.

\section{REFERENCES}

Bruggeman, J. C. 1987 Flow induced pulsations in pipe systems. Ph.D. Dissertation, Eindhoven University of Technology, Netherlands.

Bruggeman, J. C., Hirschberg, A., van Dongen, M. E. H., Wijnands, A. P. J. \& Gorter, J. 1989 Flow induced pulsations in gas transport systems: Analysis of the influence of closed side branches. ASME Journal of Fluids Engineering 111, 484-491. 
Cook, W. J. 1986 Turbulent boundary layers in oscillating flows: An experimental and computational study. NASA-CR-177109, ISU-ERI-Ames-86434-PT-1.

Crighton, D. G. 1981 Acoustics as a branch of fluid mechanics. Journal of Fluid Mechanics 106, 261-298.

DeMetz, F. C. \& Farabee, T. M. 1975 Water-flow-induced cavity resonances. Journal of the Acoustical Society of America 58 (Suppl. 1), S21.

ELDER, S. A. 1978 Self-excited depth-mode resonance for a wall-mounted cavity in turbulent flow. Journal of the Acoustical Society of America 64, 877-890.

Elder, S. A., Farabee, T. M. \& DeMetz, F. C. 1982 Mechanisms of flow-excited cavity tones at low Mach number. Journal of the Acoustical Society of America 72, 532-549.

Graf, H. R., Durgin, W. W., Erickson, D. D. \& Westhaver, R. 1988 LDV meaurements of the unsteady shear layer above a resonant cavity. 1st National Fluid Dynamics Congress, Paper AIAA-88-3547-CP.

GRAF, H. R. 1989 Experimental and computational investigation of the flow excited acoustic resonance in a deep cavity. Ph.D. Dissertation, Worcester Polytechnic Institute, Worcester, MA, U.S.A.

Howe, M. S. 1979 The influence of grazing flow on the acoustic impedance of a cylindrical wall cavity. Journal of Sound and Vibration 67, 533-544.

Michalke, A. 1984 Survey on jet instability theory. Progress in Aerospace Science 21, 159-199.

Nelson, P. A., Halliwell, N. A. \& Doak, P. E. 1981 Fluid dynamics of a flow excited resonance; Part I: Experiment. Journal of Sound and Vibration 78, 15-38.

Nelson, P. A., Halliwell, N. A. \& Doak, P. E. 1983 Fluid dynamics of a flow excited resonance; Part II: Flow acoustic interaction. Journal of Sound and Vibration 91, 375-402.

OrszaG, S. A. \& Crow, S. C. 1970 Instability of a vortex sheet leaving a semi-infinite plate. Studies in Applied Mathematics 49, 167-181.

Rockwell, D. \& NAUDASCHER, E. 1978 Review self-sustained oscillations of flow past cavities. ASME Journal of Fluids Engineering 100, 152-165.

ZIADA, S., \& Rockwell, D. 1982 Vortex-leading-edge interaction. Journal of Fluid Mechanics 118, 79-107.

ZIADA, S. 1992 Private communication.

\section{APPENDIX: NOMENCLATURE}

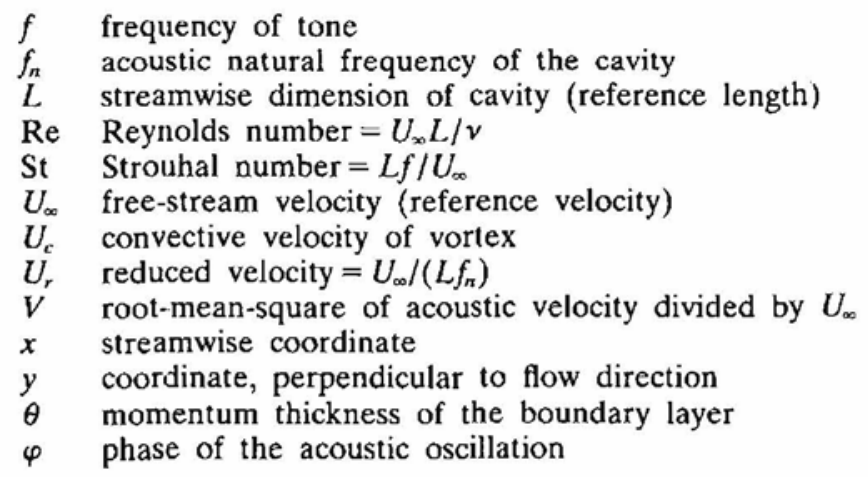

